\title{
Health Literacy in Surgical Oncology Patients: An Observational Study at a Comprehensive Cancer Center
}

\author{
Luke D. Rothermel, MD, MPH,*; Claire C. Conley, PhD 2,3,*; Anuja L. Sarode, MPH4; Michael F. Young, BS5; \\ Zulema L. Uscanga, MPH, $\mathrm{CPH}^{2}$; McKenzie Mclntyre, $\mathrm{MPH}^{2}$; Jason B. Fleming, $\mathrm{MD}^{6}$; and Susan T. Vadaparampil, $\mathrm{PhD}^{2}$
}

\begin{abstract}
Background: Low health literacy is associated with increased resource use and poorer outcomes in medical and surgical patients with various diseases. This observational study was designed to determine (1) the prevalence of low health literacy among surgical patients with cancer at an $\mathrm{NCl}$-designated Comprehensive Cancer Center (CCC), and (2) associations between health literacy and clinical outcomes. Methods: Patients receiving surgery $(\mathrm{N}=218)$ for gastrointestinal $(60 \%)$ or genitourinary cancers $(22 \%)$ or sarcomas $(18 \%)$ were recruited during their postsurgical hospitalization. Patients self-reported health literacy using the Brief Health Literacy Screening Tool (BRIEF). Clinical data (length of stay [LoS], postacute care needs, and unplanned presentation for care within 30 days) were abstracted from the electronic medical records 90 days after surgery. Multivariate linear and logistic regressions were used to examine the relationship between health literacy and clinical outcomes, adjusting for potential confounding variables. Results: Of 218 participants, 31 (14\%) showed low health literacy (BRIEF score $\leq 12$ ). In regression analyses including 212 patients with complete data, low health literacy significantly predicted $\operatorname{LoS}(\beta=-1.82 ; 95 \% \mathrm{Cl},-3.00$ to $-0.66 ; P=.002)$ and postacute care needs (odds ratio [OR], 0.25; 95\% Cl, 0.07-0.91). However, health literacy was not significantly associated with unplanned presentation for care in the 30 days after surgery (OR, $0.51 ; 95 \% \mathrm{Cl}, 0.20-1.29)$. Conclusions: This study demonstrates the prevalence of low health literacy in a surgical cancer population at a high-volume $\mathrm{NCl}$-designated $\mathrm{CCC}$ and its association with important clinical outcomes, including hospital LoS and postacute care needs. Universal screening and patient navigation may be 2 approaches to mitigate the impact of low health literacy on postsurgical outcomes.
\end{abstract}

J Natl Compr Canc Netw 2021;19(12):1407-1414 doi: 10.6004/jncen.2021.7029

1'Department of Surgery, University Hospitals Seidman Cancer Center, Cleveland, Ohio; ${ }^{2}$ Department of Health Outcomes and Behavior, Moffitt Cancer Center, Tampa, Florida; ${ }^{3}$ Department of Oncology, Georgetown University, Washington, DC; ${ }^{4}$ University Hospitals Research in Surgical Outcomes and Effectiveness Center, University Hospitals, Cleveland, Ohio; ${ }^{5}$ Morsani College of Medicine, University of South Florida, Tampa, Florida; and ${ }^{6}$ Department of Gastrointestinal Oncology, Moffitt Cancer Center, Tampa, Florida.

*These authors contributed equally to this study and are co-first authors.

\section{Background}

Health literacy consists of a set of complex and interconnected abilities that people need to function effectively in the healthcare environment. ${ }^{1-3}$ These skills impact multiple dimensions of communication and include print literacy, or the ability to read, understand, and act upon text and to locate and interpret health information in documents; and oral literacy, or the ability to speak and listen effectively about health information (eg, communicating needs to health professionals, understanding professionals' instructions). Only $12 \%$ of the US adult population is considered to have a "proficient" level of health literacy; most (53\%) have "intermediate" health literacy. ${ }^{4}$ This finding has been referred to as the "health literacy epidemic."

Low health literacy is associated with difficulty in communicating about health, including objectively poorer ability to understand and follow medical advice ${ }^{6}$ and to interpret written information in medical and surgical contexts. ${ }^{6-11}$ Low health literacy is also correlated with lower adherence to care recommendations and screening guidelines $^{6,12}$ and negatively influences clinical outcomes related to the management of chronic diseases, including asthma, ${ }^{13}$ diabetes, ${ }^{14,15}$ congestive heart failure, ${ }^{10,16,17}$ and end-stage renal disease. ${ }^{18,19}$ Finally, given the importance of health literacy for self-management after discharge, low health literacy has also been shown to be associated with a higher incidence of unplanned readmissions after hospitalization $^{20}$ and an increased rate of acute care and emergency department (ED) visits in certain patient populations. ${ }^{21}$

Compared with evidence about the relationship between health literacy and outcomes for patients with chronic medical conditions, there is a relative dearth of research on the effects of low health literacy on postoperative outcomes. A recent systematic review of health literacy in surgery identified 51 studies addressing this topic, with only 6 that investigated the association of health literacy and surgical outcomes. ${ }^{22}$ In all studies, the prevalence of low health literacy was reported in more than one-third of patients, although this was determined in heterogeneous patient populations using various assessment tools 
between studies. Low health literacy is reported to impact whether patients undergo certain surgical procedures, such as breast reconstruction after mastectomy, ${ }^{23}$ or whether they are listed for kidney transplant. ${ }^{7,24}$ In addition, low health literacy was predictive of developing minor postoperative complications in patients undergoing radical cystectomy. ${ }^{8}$ Finally, lower health literacy is independently associated with increased hospital length of stay (LoS) in patients who are undergoing major abdominal surgery. ${ }^{9}$

To our knowledge, there are no published studies focusing on the role of health literacy in complex general surgical oncology. Surgical interventions for cancers such as gastrointestinal and genitourinary malignancies and sarcomas require hospitalization and extended periods of recovery. The physical toll of these surgeries is substantial and often requires adjustments to a patient's dietary habits, functional mobility, or digestive processes, among other changes. Within the complex setting of these surgical procedures and the postoperative course, the impact of health literacy is poorly understood.

To fill this gap, the present study reports on health literacy among a sample of patients presenting for complex cancer surgery at an NCI-designated Comprehensive Cancer Center (CCC). The study aims were 2 -fold: (1) to define the prevalence of low health literacy for surgical patients with cancer at an NCI-designated CCC, and (2) to examine the associations of health literacy with clinical outcomes. We hypothesized that (1) using the Brief Health Literacy Screening Tool (BRIEF) with a cutpoint of 12, approximately $30 \%$ of surgical oncology patients would show low health literacy, defined as scores $\leq 12$ on the 4-question version of BRIEF; and (2) compared with patients with marginal or adequate health literacy, those with low health literacy would have longer hospital LoS, more postacute care needs, and more unplanned presentations for care in the 30 days after surgery.

\section{Methods}

\section{Procedures and Participants}

An observational, longitudinal, single-group design was used. All procedures were approved by the University of South Florida Institutional Review Board (protocol \#00038579). Because the BRIEF has not been used previously in the surgical literature, the data points necessary to conduct a formal power analysis were not available. Thus, the target sample size for this study (200 cases with complete data) was based on prior studies of health literacy in surgical populations. ${ }^{22}$ To achieve this goal, a convenience sample of patients presenting for care at an NCI-designated CCC was used. Recruitment occurred from March 2019 through September 2019.
Eligible participants were (1) aged $\geq 18$ years; (2) presenting for surgical treatment of primary gastrointestinal cancer, genitourinary cancer, or sarcoma; (3) admitted to the hospital for $\geq 1$ night; and (4) English speakers. Study staff reviewed lists of recent admissions and patient electronic medical records (EMRs) to identify patients meeting eligibility criteria. Eligible participants were approached by study personnel during their postsurgical inpatient stay and, if interested, provided written informed consent. After obtaining consent, study personnel assisted participants in completing a self-report survey. After survey completion, participants received a $\$ 20$ gift card in appreciation for their time and effort. Clinical data were abstracted from the EMR 90 days after surgery; thus, clinical data abstraction took place between June and December 2019.

\section{Measures}

The primary predictor of interest was health literacy. The primary outcomes of interest included hospital LoS, postacute care needs, and 30-day unplanned presentations for care postsurgery. Sociodemographic and clinical characteristics were examined as covariates.

\section{Demographic Characteristics}

Participants reported their age, sex, race/ethnicity, education level, and insurance status.

\section{Clinical Characteristics}

Medical comorbidities (Charlson-Deyo comorbidity index score $^{25}$ ), functional status (ECOG performance status ${ }^{26}$ ), and surgical complications (Clavien-Dindo grade) were abstracted from the EMRs.

\section{Health Literacy}

The 4-item $\mathrm{BRIEF}^{27}$ assesses the ability to read and discuss health-related information. This screening tool is built upon the 3-question BHLS (Brief Health Literacy Screen), ${ }^{28-33}$ with the addition of 1 question to assess oral literacy in a quick and validated measure. ${ }^{27,34}$ Responses were scored based on a 5-point Likert scale ranging from 1 (never) to 5 (always). Final scores ranged from 4 to 20, and a cutpoint of 12 is used to determine low versus marginal or adequate health literacy.

\section{Clinical Outcomes}

Data abstracted from the EMR 90 days after surgery included (1) hospital LoS (days); (2) postacute care needs, including home-based physical therapy or occupational therapy, home nursing, or discharge to a skilled nursing or rehabilitation facility (yes/no); and (3) unplanned presentations for care in the 30 days after surgery, including ED visits and/or hospital readmissions (yes/no). 


\section{Statistical Methods}

Descriptive statistics were used to examine the distribution of health literacy in this sample. Continuous variables are presented with mean [SD], and categorical variables are presented with frequency (percent). The chi-square test of association or Fisher exact test in case of cell value $<5$ was used for comparison between categorical variables. For comparing means, ANOVA was used.

In regression analyses, we combined the populations with marginal and adequate health literacy (BRIEF score $>12$ ) to highlight the impact of low health literacy (BRIEF score $\leq 12$ ) on clinical outcomes, as has been done previously. ${ }^{19,35}$ Multivariate linear regression analysis was used to examine the relationship between health literacy (low vs marginal or adequate) and hospital LoS in days (continuous). Multivariate logistic regression analysis was used to predict the odds of re-presentation to the ED/rehospitalization (yes/no) and postacute care needs (yes/no) by health literacy (low vs marginal or adequate). Initial models included sociodemographic and clinical covariates (age, sex, race/ethnicity, education level, insurance status, Charlson-Deyo scores, and Clavien-Dindo grade III or IV complications). To ensure parsimony of the final models, only those variables that were significant predictors $(P<.05)$ in the multivariate models were retained for the final model. All tests were 2 -tailed, and a $P$ value $<.05$ was considered statistically significant. All analyses were conducted using SAS 9.4 (SAS Institute Inc).

\section{Results}

Preliminary and Descriptive Analyses

We approached 255 patients, and 225 (88\%) consented to participate (Figure 1). Seven patients $(3 \%)$ were deemed ineligible after consent due to having a secondary surgery related to their cancer (ie, ileostomy takedown); thus, a total of 218 patients were included in the descriptive analyses. For regression analyses examining the relationship between health literacy and resource use, we excluded 4 outliers (Clavien-Dindo grade $\mathrm{V}, \mathrm{n}=1$; LoS $>25$ days, $\mathrm{n}=3$ ). Because 2 patients had incomplete sociodemographic data, the final analytic sample included 212 patients with complete data. For a complete description of the sample, see Table 1. Health literacy groups significantly differed on education, such that a greater proportion of patients with low health literacy had less than a high school diploma $(12.9 \%$ vs $6.1 \%$ for marginal and $2.5 \%$ for adequate). Groups also significantly differed on Charlson-Deyo score, such that a greater proportion of patients with low health literacy had $\geq 1$ comorbid condition $(61.3 \%$ vs $51.5 \%$ for marginal and $31.4 \%$ for adequate).

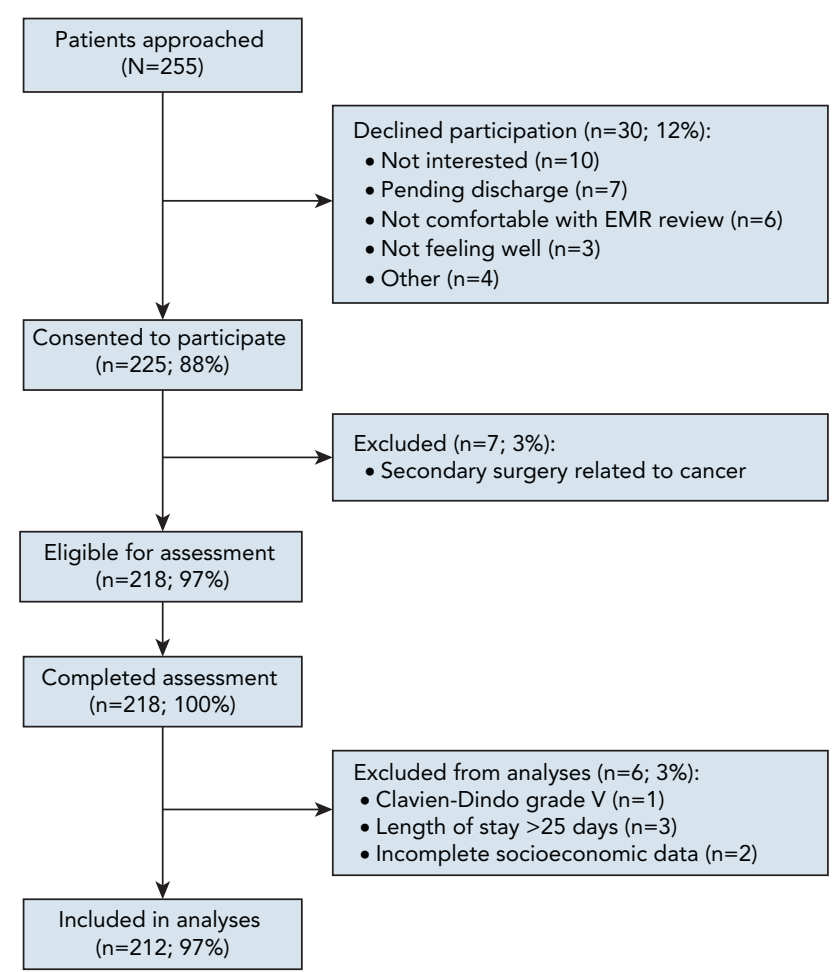

Figure 1. Study flowchart.

Abbreviation: EMR, electronic medical record.

Prevalence of Low Health Literacy

The mean [SD] BRIEF score for the sample was 16.49 [3.18]). Of 218 participants, 31 (14\%) showed low health literacy according to BRIEF scores. Marginal health literacy was identified in 66 patients (30\%), and adequate health literacy was identified in 121 (56\%).

\section{Associations With Postsurgical Outcomes}

All linear and logistic regression analyses included health literacy, age, sex, race/ethnicity, education level, insurance, Charlson-Deyo scores, and Clavien-Dindo grade III or IV complications as predictors. Results of the multivariate linear regression predicting average LoS are presented in Table 2. In the initial model, only health literacy $(P=.002)$, education level $(P=.01)$, and ClavienDindo grade III or IV complications $(P<.0001)$ significantly predicted average LoS. Thus, these 3 variables were retained in the final model for LoS. In the final model, on average, patients with marginal and adequate health literacy had significantly shorter hospital LoS than those with low health literacy $(\beta=-2.04 ; 95 \% \mathrm{CI}$, -3.19 to $-0.89 ; P=.0006$ ). In other words, on average, LoS was 2.04 days less for those with marginal or adequate health literacy than for those with low health literacy after adjusting for education and Clavien-Dindo grade III or IV complications. 


\section{Table 1. Cohort Characteristics by Health Literacy Score Category (N=218)}

\begin{tabular}{|c|c|c|c|c|}
\hline \multirow[b]{2}{*}{ Characteristic } & \multicolumn{3}{|c|}{ BRIEF Score } & \multirow[b]{2}{*}{$P$ Value } \\
\hline & $\begin{array}{c}\text { Low (6-12) } \\
n(\%)\end{array}$ & $\begin{array}{c}\text { Marginal (13-16) } \\
n(\%)\end{array}$ & $\begin{array}{c}\text { Adequate (17-20) } \\
n(\%)\end{array}$ & \\
\hline Total, $\mathrm{n}$ & 31 & 66 & 121 & \\
\hline \multicolumn{5}{|l|}{ Sociodemographic characteristics } \\
\hline Age, y, mean [SD] & $69.8[10.0]$ & $66.4[11.1]$ & $64.5[13.5]$ & .09 \\
\hline Sex & & & & .32 \\
\hline Male & $22(71.0)$ & $37(56.1)$ & $69(57.0)$ & \\
\hline Female & $9(29.0)$ & $29(43.9)$ & $52(43.0)$ & \\
\hline Race/Ethnicity & & & & $.96^{\mathrm{a}}$ \\
\hline Non-Hispanic White & $27(87.1)$ & $58(87.9)$ & $104(86.7)$ & \\
\hline Other & $4(12.9)$ & $8(12.1)$ & $17(14.1)$ & \\
\hline Education & & & & $.04^{\mathrm{a}}$ \\
\hline Less than high school diploma & $4(12.9)$ & $4(6.1)$ & $3(2.5)$ & \\
\hline High school diploma or greater & $27(87.1)$ & $62(93.9)$ & $118(97.5)$ & \\
\hline Insurance & & & & .25 \\
\hline Medicaid/Uninsured & $3(9.7)$ & $6(9.1)$ & $5(4.2)$ & \\
\hline Private/Medicare/Military & $28(90.3)$ & $60(90.9)$ & $114(95.8)$ & \\
\hline \multicolumn{5}{|l|}{ Clinical variables } \\
\hline Charlson-Deyo score & & & & $<.01$ \\
\hline 0 & $12(38.7)$ & $32(48.5)$ & $83(68.6)$ & \\
\hline$\geq 1$ & $19(61.3)$ & $34(51.5)$ & $38(31.4)$ & \\
\hline ECOG performance status & & & & $.93^{a}$ \\
\hline $0-1$ & $29(93.6)$ & $61(92.4)$ & $114(94.2)$ & \\
\hline $2-3$ & $2(6.5)$ & $5(7.6)$ & $7(5.8)$ & \\
\hline Surgery type & & & & $.56^{\mathrm{a}}$ \\
\hline Gastrointestinal & $18(58.1)$ & $36(54.6)$ & $75(62.0)$ & \\
\hline Genitourinary & $9(29.0)$ & $14(21.2)$ & $26(21.5)$ & \\
\hline Sarcoma & $4(12.9)$ & $16(24.2)$ & $20(16.5)$ & \\
\hline Clavien-Dindo grade III or IV & & & & $.12^{\mathrm{a}}$ \\
\hline Yes & $1(3.3)$ & $6(9.1)$ & $3(2.5)$ & \\
\hline No & $29(96.7)$ & $60(90.9)$ & $118(97.5)$ & \\
\hline Hospital LoS, mean (SD), d & $8.71(4.1)$ & $7.50(5.6)$ & $6.63(4.9)$ & .10 \\
\hline Re-presentation to ED/hospital & & & & .17 \\
\hline Yes & $10(32.3)$ & $12(18.2)$ & $21(17.4)$ & \\
\hline No & $21(67.7)$ & $54(81.8)$ & $100(82.6)$ & \\
\hline Postacute care needs & & & & $.02^{\mathrm{a}}$ \\
\hline Yes & $28(90.3)$ & $42(63.6)$ & $85(70.3)$ & \\
\hline Home health visit & $26(83.9)$ & $39(59.1)$ & $81(66.9)$ & \\
\hline Skilled nursing/rehabilitation facility & $2(6.5)$ & $3(4.6)$ & $4(3.3)$ & \\
\hline No & $3(9.7)$ & $24(36.4)$ & $36(29.8)$ & \\
\hline
\end{tabular}

$P$ values were derived by ANOVA, chi-square test of association, and Fisher exact test; $P<.05$ was considered statistically significant.

Abbreviations: BRIEF, Brief Health Literacy Screening Tool; ED, emergency department; LoS, length of stay.

${ }^{\text {a }}$ Fisher exact test. 


\begin{tabular}{|c|c|c|}
\hline \multicolumn{3}{|l|}{ Model 1: all covariates $\left(R^{2}=0.1951\right)$} \\
\hline Intercept & 6.140 (3.445 to 8.836$)$ & $<.0001$ \\
\hline BRIEF score, marginal or adequate (13-20) vs low (6-12) & $-1.612(-3.000$ to -0.656$)$ & .002 \\
\hline Age, y & $0.030(-0.004$ to 0.064$)$ & .080 \\
\hline Race/Ethnicity: other vs non-Hispanic White & $0.638(-0.624$ to 1.900$)$ & .320 \\
\hline Education: less than high school vs high school or greater & $2.315(0.480$ to 4.150$)$ & .014 \\
\hline Insurance: Medicaid/uninsured vs private/Medicare & -0.893 ( -2.568 to 0.782$)$ & .294 \\
\hline Charlson-Deyo score: $\geq 1$ vs 0 & $0.112(-0.717$ to 0.941$)$ & .790 \\
\hline Clavien-Dindo grade III or IV: yes vs no & 5.092 (3.016 to 7.167$)$ & $<.0001$ \\
\hline Education: less than high school vs high school or greater & $1.947(0.137$ to 3.756$)$ & .0351 \\
\hline Clavien-Dindo grade III or IV: yes vs no & 5.115 (3.033 to 7.198$)$ & $<.0001$ \\
\hline
\end{tabular}

Bold indicates statistically significant $P$ value.

Abbreviation: BRIEF, Brief Health Literacy Screening Tool.

Results of the multivariate logistic regression analyses predicting the odds for re-presentation to the ED/ rehospitalization are presented in Table 3 . In the initial model, none of the specified predictors were significantly associated with re-presentation to the ED/ rehospitalization.

Results of the multivariate logistic regression analyses predicting the odds for postacute care needs are presented in Table 4. In the initial model, only health literacy (odds ratio $[\mathrm{OR}$ ], 0.25; 95\% CI, 0.07-0.91), age (OR, 1.05; 95\% CI, 1.02-1.08), and sex (OR, 2.10; 95\% CI, 1.06-4.18) significantly predicted the odds for postacute care needs. Thus, these 3 variables were retained in the final model for the odds of postacute care needs. In the final model, patients with marginal and adequate health literacy were $77 \%$ less likely to need postacute care than those with low health literacy (OR, 0.23; 95\% CI, 0.07-0.82) after adjusting for age and sex.

\section{Discussion}

Low health literacy is associated with increased resource use $^{6,12,20,21}$ and poorer clinical outcomes ${ }^{6,10,11}$ in medical and surgical patients with various diseases. Despite the demonstrated importance of health literacy in the perioperative period, ${ }^{35,36}$ the role of health literacy in complex surgical oncology has not previously been delineated. Because surgery for cancer is especially complex in terms of shared decision-making, perioperative instructions, and

\begin{tabular}{|c|c|}
\hline Variable & OR $(95 \% \mathrm{Cl})$ \\
\hline BRIEF score: marginal or adequate (13-20) vs low (6-12) & $0.508(0.200-1.291)$ \\
\hline Age, $y$ & $1.006(0.976-1.038)$ \\
\hline Education: less than high school vs high school or greater & $0.659(0.121-3.587)$ \\
\hline Insurance: Medicaid/uninsured vs private/Medicare & $3.033(0.873-10.542)$ \\
\hline Charlson-Deyo score: $\geq 1$ vs 0 & $1.527(0.740-3.150)$ \\
\hline Clavien-Dindo grade III or IV: yes vs no & $2.768(0.324-23.653)$ \\
\hline
\end{tabular}

Abbreviations: AUC, area under the curve; BRIEF, Brief Health Literacy Screening Tool; ED, emergency department; OR, odds ratio. 


\begin{tabular}{|l|l|}
\hline Table 4. Logistic Regression Analysis for Factors Predicting Postacute Care Needs (n=212) \\
\hline Variable & OR (95\% CI) \\
\hline \begin{tabular}{l} 
Model 1: all covariates (AUC, 0.713) \\
\hline BRIEF score: marginal or adequate (13-20) vs low (6-12)
\end{tabular} & $\mathbf{0 . 2 5 4}(0.071-0.908)$ \\
\hline Age, y & $\mathbf{1 . 0 5 2}(1.023-1.082)$ \\
\hline Sex: female vs male & $\mathbf{2 . 1 0 1}(1.058-4.175)$ \\
\hline Race/Ethnicity: other vs non-Hispanic White & $0.716(0.272-1.884)$ \\
\hline Education: less than high school vs high school or greater & $2.879(0.499-16.605)$ \\
\hline Insurance: Medicaid/uninsured vs private/Medicare & $1.223(0.279-5.363)$ \\
\hline Charlson-Deyo score: $\geq 1$ vs 0 & $0.937(0.486-1.807)$ \\
\hline Clavien-Dindo grade III or IV: yes vs no & $2.768(0.324-23.653)$ \\
\hline Model 2: parsimonious model (AUC, 0.689) & $\mathbf{0 . 2 3 2}(0.065-0.821)$ \\
\hline BRIEF score: marginal or adequate (13-20) vs low (6-12) & $\mathbf{1 . 0 4 9}(1.022-1.077)$ \\
\hline Age, y & $\mathbf{1 . 9 7 0 ~ ( 1 . 0 1 7 - 3 . 8 1 6 ) ~}$ \\
\hline Sex: female vs male & \\
\hline
\end{tabular}

Bold indicates statistically significant $P$ value.

Abbreviations: AUC, area under the curve; BRIEF, Brief Health Literacy Screening Tool; OR, odds ratio.

the postoperative course, there is an urgent need to understand the role of health literacy in outcomes after cancer surgeries. The present study fills this gap. Our data show that only a small proportion $(14 \%)$ of patients presenting for complex cancer surgery at a high-volume NCI-designated CCC had low health literacy. Nonetheless, lower health literacy was related to increased resource use in the form of hospital LoS and postacute care needs. Taken together, these data have clinical implications for the identification and management of patients with low health literacy.

Our first hypothesis, that approximately $30 \%$ of participants would show low health literacy, was not supported. The incidence of low health literacy in this cohort (14\%) is lower than the previously reported average rate in other surgical cohorts $(\sim 33 \%){ }^{22}$ Because $12 \%$ of the patients approached refused participation, it is possible that these patients may have disproportionately represented the low health literacy group, thereby skewing our data. Alternatively, this lower rate of low health literacy may reflect differences in the types of patients who present to regional CCCs; across most cancer diagnoses, patients are more likely to be treated at NCI-designated CCCs if they are non-Hispanic White, are privately insured, and have high socioeconomic status. ${ }^{37}$ These characteristics have also been associated with higher health literacy. ${ }^{6,38-40}$ Seeking treatment at a stand-alone NCI-designated CCC often requires a high level of patient engagement to obtain a referral or navigate care between medical systems. Thus, higher levels of health literacy may be necessary to establish care at these institutions.

Although small numbers of patients in this sample showed low health literacy, BRIEF scores were still related to longer hospital LoS and greater postacute care needs when accounting for the sociodemographic and clinical variables that were significantly related to outcomes of interest. Even at an NCI-designated CCC that has incorporated perioperative interventions that reduce LoS after surgery, ${ }^{41}$ we still observed differences in clinical outcomes by level of health literacy. Thus, our second hypothesis was partially supported. We did not observe any relationship between BRIEF scores and unplanned presentation for care within 30 days after surgery. This contradicts the findings in certain medical conditions showing that low health literacy is related to higher incidence of unplanned readmissions after hospitalization $^{20}$ and an increased rate of acute care and ED visits ${ }^{21}$ in the 30 days after surgery. There are several potential explanations for our null findings. First, our results could be confounded in part by the source of data on unplanned presentation for care. These data were abstracted from the institutional EMRs, which may not capture readmissions and ED visits at outside facilities. Thus, the data presented here may underestimate unplanned re-presentations for care in the 30 days after surgery. In addition, our patient population included patients who underwent complex surgeries for multiple types of cancers (gastrointestinal, genitourinary, and sarcoma), for whom different degrees of morbidity and postacute care needs would be expected, depending on the surgery performed. Although different trends existed for resource use by cancer types, the low number of patients in these independent groups precluded subgroup analyses. Therefore, this heterogeneity may limit the sensitivity of our analysis.

These data have important system-level implications. Specifically, screening for low health literacy in 
the postsurgical setting was feasible; we did not observe patient unwillingness to participate due to stress, postoperative pain, or medication-related impairment. Other studies that have reviewed health literacy postoperatively have done so in the outpatient clinic setting. ${ }^{7,23,42}$ Therefore, the feasibility of health literacy screening during this postoperative admission is a novel contribution to the literature.

Although universal health literacy screening has been criticized for its potential to result in negative profiling of patients through perceived or actual stigmatization, ${ }^{43-48}$ the present study suggests that screening for health literacy may allow a targeted intervention approach to reduce the clinical impact of low health literacy. This aligns with recent recommendations for the use of universal health literacy screening in addition to universal precautions (eg, plain language and teach-back communications for all patients) as best practices for managing patients with low health literacy. ${ }^{49}$ With multiple studies demonstrating increased resource use by patients with low health literacy (ie, LoS), a proactive realignment of resources (eg, high-touch interaction with the clinical team, patient navigation, social work, home care assistance) earlier in the perioperative period could help these patients adhere to standard treatment courses and mitigate the impact of low health literacy on clinical outcomes.

Once patients with low health literacy are identified, evidence-based interventions could be used to improve the postoperative outcomes and resource requirements of this population. ${ }^{50}$ For example, intensive selfmanagement interventions (eg, tailored educational materials about disease management and scheduled telephone follow-up) have been shown to reduce ED visits and hospitalizations. ${ }^{51,52}$ However, to our knowledge, these interventions have not been tested in the surgical oncology context. Future studies are needed to assess whether the positive intervention results observed in other populations would translate to this setting. In addition, there has been a recent push for the integration of nurse navigators in the postsurgical setting. ${ }^{53,54}$ Emerging data show that nurse navigation interventions can reduce hospital LoS and postsurgical readmissions. ${ }^{55}$ Future research should examine the potential of navigation interventions specifically for surgical patients with low health literacy.

Strengths of our study include the direct clinical applicability and the novel application of health literacy screening in the postsurgical setting. Furthermore, this study is among the first to examine health literacy in the complex surgical oncology setting. To our knowledge, this is the first evaluation to focus on health literacy in surgical patients at a high-volume, freestanding, NCI-designated CCC. We also used an established measure to assess health literacy: the BRIEF. ${ }^{27}$
Several limitations must also be acknowledged. Data from a single institution may not be generalizable across all surgical practice settings. We had limited ability to examine the potential confounding role of sociodemographic characteristics due to the homogeneous sample (ie, $87 \%$ non-Hispanic White patients, 95\% high school diploma or higher education level, 93\% insured). Furthermore, this homogeneous sample reduces the generalizability of results to diverse patient populations. EMR data exclusive to our institution may underrepresent ED visits and readmissions if they occurred at outside facilities. The convenience sample of patients presenting for surgery may be subject to selection bias, and self-reported health literacy may be subject to demand characteristics and social desirability. Lastly, there may be additional predictors of resource use (eg, psychologic symptoms, social support, quality of or satisfaction with care) that were not assessed in this study.

\section{Conclusions}

Low health literacy adversely affects clinical outcomes and resource use in medical and surgical patients. This pilot study demonstrates the prevalence of low health literacy in a surgical cancer population at a high-volume NCIdesignated CCC and its association with important clinical outcomes, including hospital LoS and postacute care needs. Health literacy is a patient-level determinant of health that should be considered among other biologic, psychologic, social, and health system determinants of health when evaluating patients' ability to navigate their complex medical care.

\section{Acknowledgments}

We wish to thank Sarah Zhu and Hansel Baez for their assistance in identifying eligible participants.

Submitted December 26, 2020; final revision received February 8, 2021; accepted for publication February 17, 2021.

Author contributions: Study design: Rothermel, Conley. Recruited and consented participants and administered surveys: Rothermel, Conley, Young, Uscanga, Mclntyre. Patient chart review: Rothermel, Young. Data analysis: Conley, Sarode. Study oversight and guidance: Fleming, Vadaparampil. Writing - original draft: Rothermel, Conley, Sarode. Writing - review and editing: All authors.

Disclosures: The authors have disclosed that they have not received any financial consideration from any person or organization to support the preparation, analysis, results, or discussion of this article.

Funding: Research reported in this publication was supported by the $\mathrm{NCl}$ of the NIH under award number T32CA090314 (S.T. Vadaparampil). This work was also supported by funding from Moffitt Cancer Center to Dr. Rothermel (Junior Scientist Partnership Fund; L.D. Rothermel and C.C. Conley, principal investigators).

Disclaimer: The content is solely the responsibility of the authors and does not necessarily represent the official views of the NIH.

Correspondence: Luke D. Rothermel, MD, MPH, Department of Surgery, University Hospitals Seidman Cancer Center, Lakeside 7010, 11100 Euclid Avenue, Cleveland, OH 44139. Email: luke.rothermel@uhhospitals.org 


\section{References}

1. Berkman ND, Sheridan SL, Donahue KE, et al. Health literacy interventions and outcomes: an updated systematic review. Evid Rep Technol Assess (Full Rep) 2011;199:1-941.

2. Sørensen K, Van den Broucke S, Fullam J, et al. Health literacy and public health: a systematic review and integration of definitions and models. BMC Public Health 2012;12:80.

3. Peerson A, Saunders M. Health literacy revisited: what do we mean and why does it matter? Health Promot Int 2009;24:285-296.

4. Kutner M, Greenburg E, Jin Y, et al. The Health Literacy of America's Adults: Results From the 2003 National Assessment of Adult Literacy (NCES 2006-483). Washington, DC: National Center for Education Statistics, US Department of Education; 2006.

5. Davis TC, Wolf MS. Health literacy: implications for family medicine. Fam Med 2004;36:595-598.

6. Berkman ND, Sheridan SL, Donahue KE, et al. Low health literacy and health outcomes: an updated systematic review. Ann Intern Med 2011;155:97-107.

7. Kazley AS, Hund JJ, Simpson KN, et al. Health literacy and kidney transplant outcomes. Prog Transplant 2015;25:85-90.

8. Scarpato KR, Kappa SF, Goggins KM, et al. The impact of health literacy on surgical outcomes following radical cystectomy. J Health Commun 2016;21: 99-104.

9. Wright JP, Edwards GC, Goggins K, et al. Association of health literacy with postoperative outcomes in patients undergoing major abdominal surgery. JAMA Surg 2018;153:137-142.

10. McNaughton CD, Cawthon C, Kripalani S, et al. Health literacy and mortality: a cohort study of patients hospitalized for acute heart failure. J Am Heart Assoc 2015;4:e001799.

11. Zhang NJ, Terry A, McHorney CA. Impact of health literacy on medication adherence: a systematic review and meta-analysis. Ann Pharmacother 2014:48:741-751.

12. Oldach BR, Katz ML. Health literacy and cancer screening: a systematic review. Patient Educ Couns 2014;94:149-157.

13. Mancuso CA, Rincon M. Impact of health literacy on longitudinal asthma outcomes. J Gen Intern Med 2006;21:813-817.

14. Osborn CY, Cavanaugh K, Kripalani S. Strategies to address low health literacy and numeracy in diabetes. Clin Diabetes 2010;28:171-175.

15. Schillinger D, Bindman A, Wang F, et al. Functional health literacy and the quality of physician-patient communication among diabetes patients. Patient Educ Couns 2004;52:315-323.

16. Peterson PN, Shetterly SM, Clarke CL, et al. Health literacy and outcomes among patients with heart failure. JAMA 2011;305:1695-1701.

17. Wu JR, Holmes GM, DeWalt DA, et al. Low literacy is associated with increased risk of hospitalization and death among individuals with heart failure. J Gen Intern Med 2013;28:1174-1180.

18. Cavanaugh $\mathrm{KL}$, Osborn CY, Tentori F, et al. Performance of a brief survey to assess health literacy in patients receiving hemodialysis. Clin Kidney J 2015 8:462-468.

19. Dageforde LA, Box A, Feurer ID, et al. Understanding patient barriers to kidney transplant evaluation. Transplantation 2015;99:1463-1469.

20. Bailey SC, Fang G, Annis IE, et al. Health literacy and 30-day hospital readmission after acute myocardial infarction. BMJ Open 2015;5:e006975.

21. Mitchell SE, Sadikova E, Jack BW, et al. Health literacy and 30-day postdischarge hospital utilization. J Health Commun 2012:17:325-338.

22. Chang ME, Baker SJ, Dos Santos Marques IC, et al. Health literacy in surgery. Health Lit Res Pract 2020;4:e46-65.

23. Winton LM, Nodora JN, Martinez ME, et al. Factors associated with surgical management in an underinsured, safety net population. Surgery 2016;159:580-590.

24. Warsame $F$, Haugen $C E$, Ying $H$, et al. Limited health literacy and adverse outcomes among kidney transplant candidates. Am J Transplant 2019;19:457-465.

25. Charlson ME, Pompei P, Ales KL, et al. A new method of classifying prognostic comorbidity in longitudinal studies: development and validation. J Chronic Dis 1987;40:373-383.

26. Oken MM, Creech $\mathrm{RH}$, Tormey DC, et al. Toxicity and response criteria of the Eastern Cooperative Oncology Group. Am J Clin Oncol 1982;5:649-656.

27. Haun J, Noland-Dodd V, Varnes J, et al. Testing the BRIEF Health Literacy Screening Tool. Fed Pract 2009:26:24-31.

28. Chew LD, Bradley KA, Boyko EJ. Brief questions to identify patients with inadequate health literacy. Fam Med 2004;36:588-594.
29. Chew LD, Griffin JM, Partin MR, et al. Validation of screening questions for limited health literacy in a large VA outpatient population. J Gen Intern Med 2008;23:561-566.

30. Wallace LS, Cassada DC, Rogers ES, et al. Can screening items identify surgery patients at risk of limited health literacy? J Surg Res 2007;140:208-213.

31. Wallace LS, Rogers ES, Roskos SE, et al. Brief report: screening items to identify patients with limited health literacy skills. J Gen Intern Med 2006 21:874-877.

32. Sarkar U, Schillinger D, López A, et al. Validation of self-reported health literacy questions among diverse English and Spanish-speaking populations. J Gen Intern Med 2011;26:265-271.

33. Cawthon C, Mion LC, Willens DE, et al. Implementing routine health literacy assessment in hospital and primary care patients. Jt Comm J Qual Patient Saf 2014:40:68-76.

34. Haun JN, Patel NR, French DD, et al. Association between health literacy and medical care costs in an integrated healthcare system: a regiona population based study. BMC Health Serv Res 2015;15:249.

35. Zite NB, Wallace LS. Use of a low-literacy informed consent form to improve women's understanding of tubal sterilization: a randomized controlled trial. Obstet Gynecol 2011;117:1160-1166.

36. Chew LD, Bradley KA, Flum DR, et al. The impact of low health literacy on surgical practice. Am J Surg 2004;188:250-253.

37. Wolfson JA, Sun CL, Wyatt LP, et al. Impact of care at comprehensive cancer centers on outcome: results from a population-based study. Cancer 2015:121:3885-3893.

38. Wolf MS, Gazmararian JA, Baker DW. Health literacy and functional health status among older adults. Arch Intern Med 2005;165:1946-1952.

39. von Wagner C, Knight K, Steptoe A, et al. Functional health literacy and health-promoting behaviour in a national sample of British adults. J Epidemiol Community Health 2007;61:1086-1090.

40. Baker DW, Gazmararian JA, Williams MV, et al. Functional health literacy and the risk of hospital admission among Medicare managed care enrollees. Am J Public Health 2002;92:1278-1283.

41. Broman $\mathrm{KK}$, Baez H, Mihelic $\mathrm{E}$, et al. Combined use of advanced practice providers and care pathways reduces the duration of stay after surgery for gastrointestinal malignancies. Surgery 2021;169:852-858.

42. Serper M, Patzer RE, Reese PP, et al. Medication misuse, nonadherence, and clinical outcomes among liver transplant recipients. Liver Transpl 2015 21:22-28.

43. Paasche-Orlow MK, Wolf MS. Evidence does not support clinical screening of literacy. J Gen Intern Med 2008;23:100-102.

44. Parikh NS, Parker RM, Nurss JR, et al. Shame and health literacy: the unspoken connection. Patient Educ Couns 1996;27:33-39.

45. Wolf MS, Williams MV, Parker RM, et al. Patients' shame and attitudes toward discussing the results of literacy screening. J Health Commun 2007 12:721-732.

46. Garg A, Boynton-Jarrett R, Dworkin PH. Avoiding the unintended consequences of screening for social determinants of health. JAMA 2016;316:813-814.

47. Blair IV, Steiner JF, Havranek EP. Unconscious (implicit) bias and health disparities: where do we go from here? Perm J 2011;15:71-78.

48. Schlichting JA, Quinn MT, Heuer LJ, et al. Provider perceptions of limited health literacy in community health centers. Patient Educ Couns 2007:69:114-120.

49. Hadden KB, Kripalani S. Health literacy 2.0: integrating patient health literacy screening with universal precautions. Health Lit Res Pract 2019;3:e280-285.

50. Walters R, Leslie SJ, Polson R, et al. Establishing the efficacy of interventions to improve health literacy and health behaviours: a systematic review. BMC Public Health 2020;20:1040.

51. Sheridan SL, Halpern DJ, Viera AJ, et al. Interventions for individuals with low health literacy: a systematic review. J Health Commun 2011;16:30-54

52. Schaffler J, Leung K, Tremblay S, et al. The effectiveness of self-management interventions for individuals with low health literacy and/or low income: a descriptive systematic review. J Gen Intern Med 2018;33:510-523.

53. DeGrace L. The role of the surgical nurse navigator: a case scenario. Can Oncol Nurs J 2018;28:322-323.

54. Burch J. Enhanced recovery and nurse-led telephone follow-up post surgery. Br J Nursing 2012;21:S24-29.

55. Watkins EJ. Evaluation of a Cardiac Surgery Nurse Navigator Quality Improvement Program. Dissertation. Walden University; 2021. Accessed July 21, 2021. Available at: https://scholarworks.waldenu.edu/ dissertations/10012/ 